\title{
Paracelsus, die Bienen und die «Signaturen»
}

\author{
Von Hubert Erhard, Adelholzen (Oberbayern)
}

In den wenigen Seiten, die Paragelsus den Blumen und den Bienen widmet, und die vor allem im 2. Band, S. 193 ff., der von Sudноғf besorgten Gesamtausgabe stehen, steckt manches, was vom Standpunkt der allgemeinen Biologie uns heute noch fesselt.

Nach Paracelsus ist die «prima materia des Honigs» die «Süße der Erde», welche wiederum ein Teil der gesamten «prima materia» der Erde ist. In ihr ist «alles vorhanden, was zu den Gewächsen gehört». Aus dieser prima materia der Erde zieht die Pflanze «ein jegliches, was ihr gehört», auch die Süße, durch die «anziehende Kraft der Gewächse». Wie sich nun in der Retorte des Alchimisten «allemal das Gröbste zu Boden setzt, und das Reinere und Subtilere in die Höhe treibt», so setze sich dreierlei «Honig» in einem Gewächs ab: «das Unterste, das Mittelste und das Oberste. Das Oberste, das suchen die Immen; das liegt in den Blumen in der Blüte und in den Knospen.» Aus den Blumen «däuen» d.h. essen und verdauen die Bienen. Das Verdaute werfen sie durch «Ausscheidungsöffnungen» wieder als Honig und Wachs aus.

Es war natürlich Paracelsus bekannt, daß sich die Beschaffenheit des Honigs nach der Tracht richtet. Blüten wild wachsender Bäume würden «grobe, bäuerliche Speise» den Bienen geben, wobei wiederum ein Unterschied sei, ob es sich um Lindenblüten, Buchenblüten oder Eichenblüten handle. Dagegen würden gezüchtete Blumen, wie Rosen, Lilien, «den edelsten Honig und Wachs, gut zu süßem Met und zu subtilen Pflastern, geben ». Im Bienenstock befänden sich zwei Stände : «edle und unedle» Bienen; durch «Heirat zwischen adligen und bäurischen Immen» entstünden die «bürgerlichen» Bienen. «Die sich in der Wilde behelfenden » Immen «sind grober, bäurischer Art; die geben einen harten, starken, groben Honig, kernhaft und nährhaft ${ }^{1}$. Denn die Bauern sind im Volk die ersten und bleiben auch die letzten.» Die «adeligen» Immen gehen an die «gezierten Blumen»; in den «Bürgern» "komme Grobes und Reines zusammen»; das wird ein «temperierter» (ausgeglichener) «Honig und Wachs». «Dieser Unterschied» (in der Beschaffenheit des Honigs) «soll im Gedächtnis behalten werden von den Ärzten, denn» (danach) «teilen sich auch die Arzneien, die von ihnen gemacht werden.»

1 Er hat schon als Knabe wohl den Schweizer Gebirgshonig bei Einsiedeln kennengelernt. Man denke etwa an den harten, schneidbaren weißen Gebirgshonig, von dem heute der weiße Honig von Sanetsch der bekannteste ist. 
Des Paracelsus Auffassung vom Seelenleben der Tiere: Ihr höchstes Gut sei «das ihnen wohl tut zu ihrer Nahrung»; dem Wolf sei höchstes Gut «Stehlen und Rauben», den Immen «ein fröhliches Wabenfüllen mit Wachs und mit Honig». «Gott, der ihnen gegeben hat, Künstler zu sein, und zu heißen, hat ihnen auch das Regiment gegeben.» Sie sind «mit Ordnung unter sich selbst begabt, nicht nach der Ordnung des Gesetzes der Menschen, sondern nach der Ordnung ihrer angeborenen Natur.» Sie haben dazu ihren «König» wie «die Schnaken ihren Vorflieger» und andere Tiere «ihren Obersten», «so haben sie es auch und besser als die Tiere alle; denn sie sind dem Menschen am gleichesten». «Es erweist sich durch Erfahrenheit, daß keinerlei Tier erscheint, das dem Menschen in allem so gleich ist und ein so vernünftiges Tier ist.» «Und der Mensch kann so wenig ihre Geheimnisse erfahren, das ist die Mysteria ihrer Heimlichkeit, so wenig sie die des Menschen ergründen können. Und so wenig er ihnen das ihrige nachtun kann, so wenig sie ihm auch.» ${ }^{2}$

Paracelsus versucht also Vorgänge der Natur auf natürliche Weise zu erklären, ist aber andererseits doch noch ganz in seiner Zeit befangen durch die Lehre von den «Signaturen» ${ }^{3}$. Nach Paracelsus ist «jedliches Ding» «elementisch und firmamentisch». Unter «elementisch» versteht er die Eigenbildung des Körpers; beim Organismus also den «inneren Bildungstrieb» (Goethe). Unter «firmamentisch» die Beziehung nicht nur zur näheren, sondern auch zur weiteren Umwelt, ja zum Weltall; im erweiterten Sinn also «die äußeren Umstände» (Goethe). Aus der äußeren Gestalt müsse sich deshalb nach Paracelsus die Bestimmung des Lebewesens im Kosmos erkennen lassen; «denn Gott hat im Anfang all diese Dinge fleißig unterschieden und keinem wie dem andern eine Gestalt und Form gegeben, sondern einem jeden eine Schelle angehängt, wie man sagt: man erkennt den Narren an der Schelle. Also sollt ihr nun auch die Kräuter und Wurzeln an den Schellen erkennen.» Nun erfolgt der Sprung vom naturwissenschaftlichen Denken zur Magie, indem er fortfährt: «Durch die Kunst Chiromantia, Physiognomia und Magia ist es möglich, gleich von Stund an, nach dem äußerlichen Ansehen, eines jeden Krautes und jeder Wurzel Eigenschaft und Tugend zu erkennen an seinen Signa, an seiner Gestalt, Form und Farbe, und das bedarf keiner Probierung oder langen Erfahrung.» Um der «Süße» willen wachse der Thymian, seine Gestalt sei gewissermaßen ein Sinnbild für diese «Süße». - Und nun der Gedankensprung von der Pflanze zum Menschen: Es gebe eine Krankheit des Menschen, die sich in ihrer Form auf der menschlichen Haut wie der Thymian ausbreitet, «der aus seinem Stengel einen großen Kolben» (eine dicke Pfahlwurzel) «hinaustreibt, welcher sich mit kleinen Verzweigungen ausbreitet,

2 Bd. 14, S. $117 \mathrm{ff.} \mathrm{:} \mathrm{«Liber} \mathrm{de} \mathrm{nymphis».}$

3 Bd.1, S. 326 ff.; Bd.2, S. 86 ff.; Bd.13, S. 376; Bd.14, S.182; Bd. 11, S. 373-403. 
die an ihren Enden» (zu Blüten) «wieder anschwellen» ${ }^{4}$. "Wie die Immen auf dem Thymian sitzen und seine Süße saugen, so ist auch eine solche Süße in diesen» (Menschen-)«Geschlechtern thymischer Art, so daß die Läuse ihrer Süße zulaufen.» «Darum wisset, daß» (diese Krankheiten) «dem Thymian nach geheißen werden» (Thimia), «denn eine Art ist es, diese und jener. Nun steht ihre Heilung allein in dem Punkt, daß ihre Süße genommen wird».

Paracelsus lehrt, Pflanzensigna, die dem Menschen besonders auffielen, gäben dem Menschen einen Fingerzeig, daß die betreffende Pflanze für ihn ein spezifisches Heilmittel sei. Weil die Wurzelknolle der Orchidee, des «Knabenkrauts», einem Hodenpaar ähnlich ist, «bringe sie Männern ihre verlorene Mannheit wieder»; weil die Distel sticht, sei sie ein Mittel gegen Seitenstechen; weil das Schöllkraut einen gelben Saft hat, helfe es gegen Gelbsucht; das Johanniskraut wegen seiner durchlöchert aussehenden Blätter gegen Stichwunden; die Päonie sei ein Mittel gegen Gehirnschlag, weil ihr Griffel hirnförmig ist; der einem Kettenpanzer ähnliche Wurzelstock des Allermannsharnisch schütze gegen Hieb und Stich; die (schwer zu bestimmende) Pflanze «Syderica» mit schlangenähnlicher Blattzeichnung gegen Schlangengift ${ }^{5}$. Und der gleiche Paracelsus, der sein Leben lang gegen den Autoritätsglauben in der Medizin gekämpft hat, der sich nur auf die eigene Erfahrung und Erprobung verlassen hat, übernimmt solche Vorstellungen seiner Zeit und schreibt: «Und das bedarf keiner Probierung und Erfahrung. »

C. G. Jung schreibt ${ }^{6}$ : «Das Studium der Schriften des Paracelsus ist erschwert durch viele Unklarheiten und Widersprüche ${ }^{7}$ und namentlich durch die von Paracelsus reichlich verwendete Arcanterminologie.» Ein Beispiel für dieses Hineingeheimnissen auf Grund einer Zufallsbeobachtung: Die Ahnung des Paracelsus, daß die Wegwarte, die ihre Blüten immer der Sonne zukehrt, deshalb,

4 Es handelt sich um das Fleckfieber, Typhus exanthematicus, die «Läusesucht». «Um den 6. Krankheitstag pflegt das Exanthem am Rumpf auszubrechen und sich auch auf die Extremitäten, Fußsohlen und Handteller auszubreiten.» (SEIFERT und MüLLER, Taschenbuch der medizinisch-klinischen Diagnostik, 18. Auflage, S.10.).

5 Bd.13, S. 377.

6 C. G. Jung, Paracelsus als Arzt, in Verh. Schweiz. Naturf. Gesell. 1941, 207, auch in C. G. Jung, Paracelsica, Zürich.

7 Dagegen ist es kein Widerspruch, sondern nur eine Einschränkung, wenn er gerade in derjenigen Schrift, in der er dem Autoritätsglauben seine eigene Erfahrung besonders deutlich gegenüberstellt, im Kommentar der Aphorismen des Hippokrates, schreibt: «Wir haben Experienz (= Erfahrung) und doch nicht ganz ... Wir wissen, daß die Arzneimittel einen Tag wirken, den andern nicht ... Wo wir verzweifeln, da helfen sie, und wo wir hoffen, da verlassen sie uns. Denn wir haben die Ursachen (der Erkrankungen) nicht in unserer Hand, und das ist (der Grund unserer) fallax (Täuschung) ... Vertrau Deiner Erfahrenheit nichts in der Natur, denn sie ist in eines Arztes Hand wie ein Herz einer Frau in eines Buhlen Hand ...» 
solange die Sonne scheint, mehr «Kraft» als im Dunkeln habe, ist richtig (wir würden sagen: mehr Chlorophyll und Vitamine enthält). Paracelsus aber fährt fort: «Aus welcher Ursache meinst Du, daß ihre Wurzel sich nach sieben Jahren in eines Vogels Gestalt verwandle, und was zeigt die Magie davon an?» (In Wirklichkeit verzweigt sich die Wurzel der Wegwarte nach sieben Jahren ganz verschieden; zufällig kann sie auch einmal einem Vogel ähnlich sehen.) Paracelsus aber schreibt weiter: «Weißt Du das, so schweig still dazu und sage keinem Spötter was davon; weißt Dus aber nicht, so lerne es wissen und frage ihm nach und schäme Dich nicht zu fragen. Denn wer fragt, geht nicht irr und tut nicht unrecht, wie man sagt. Darum lerne, lerne, frage, frage und schäme Dich nicht!»

Für den in der Signaturenlehre Befangenen waren diese Zeichen Offenbarungen Gottes, und, wenn der Mensch sie nicht verstand, so war dies eben die Schuld des Menschen, ein Ansporn, weiter darüber zu forschen.

Hans Fischer schreibt ${ }^{8}$ : "Als Bauernbub hat Paracelsus im rauhen Waldtal der Sihl den Duft der bunten Alpenwiesen in sich aufgenommen.» Wir wissen wenig über seine Kindheit, doch schildert KolBENHEYER ${ }^{9}$, seiner dichterischen Intuition fogend, wie sich wohl der Knabe unbefangen in die Betrachtung der Natur und des Menschen vertieft habe. Hätte nicht der unverbildete Jüngling das Nächstliegende entdecken können, daß nämlich Form, Farbe, Duft der Blumen - sozusagen ihre «Schellen» - nicht des Menschen wegen, sondern der die Blüten besuchenden Insekten wegen geschaffen sind? Doch mit zunehmender «Reife» unterlag selbst er, der auf medizinischem Gebiet zeitlebens den Autoritätsglauben seiner Zeit bekämpft hat, der Befangenheit und dem Vorurteil seiner Zeit als Naturforscher. So hat erst 1793 Chr. K. Sprengel «das entdeckte Geheimnis der Natur im Bau und in der Befruchtung der Blumen» geklärt.

Dadurch erst ist der Weg für eine vorurteilsfreie Naturforschung freigemacht worden, die mit der Morphologie und Physiologie der Blüte und des dazu gehörigen, die Befruchtung vermittelnden Insekts beginnt, über die Sexualität der staatenbildenden Insekten durch Dzierzon hinausführt und schließlich in der Erforschung der Psychologie derselben durch KARL von Frisch in seiner Sprache der Bienen ihren Abschluß findet.

Bekannt ist, daß der Glaube, alles sei des Menschen wegen geschaffen, die Erde müsse deshalb Mittelpunkt der Welt sein, der Ausbreitung der Lehre des CoperNIKUS entgegenstand. Aber es wird zu wenig beachtet, daß auch in der Biologie die anthropozentrische Auffassung jahrhundertelang ein Hemmschuh gewesen ist. Welche Entwicklung hätte die Naturforschung im Laufe der Geschichte genommen, wenn sie, frei von solchen selbst auferlegten Fesseln, sich nur der Erforschung der Wahrheit gewidmet hätte!

8 Hans Fischer, Der junge Paracelsus, in Verh. Schweiz. Nat.Ges.1941, S. 209.

9 E.G.Kolbenheyer, Paracelsus, I.Teil: Die Kindheit des Paracelsus, München 1923. 